\title{
HACIA LA MEDICINA PERSONALIZADA: IMPLICANCIAS DE LAS CIENCIAS BÁSICAS Y LAS “ÓMICAS” EN LA PRÁCTICA CLÎNICA
}

\author{
TOWARDS PERSONALIZED MEDICINE: IMPLICATIONS OF BASIC SCIENCES ANDTHE \\ "OMICS"IN CLINICAL PRATICE
}

Heinner Guio 1,a

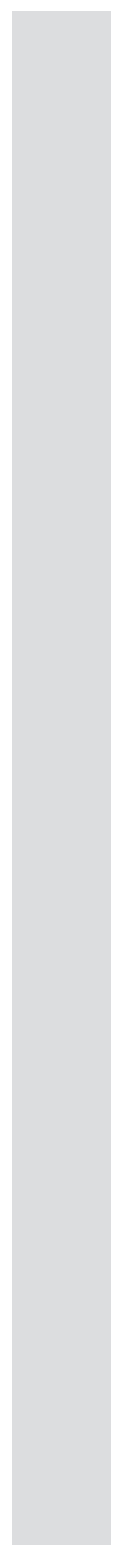

\begin{abstract}
El proceso de aplicación de los hallazgos en ciencias básicas en las intervenciones clínicas, cada vez está siendo más difundido por las instituciones académicas e institutos nacionales de investigación debido al impacto que tendrán en el desarrollo de la medicina personalizada. Las ciencias básicas constituyen la base fundamental de los procesos de desarrollo científico y tecnológico, sin los cuales es impensable la inserción de nuestro país en las dinámicas globales de innovación en salud. La producción de métodos de diagnóstico de muy alta tecnología, el desarrollo de biomarcadores de seguimiento en enfermedades trasmisibles y no trasmisibles debería ser elegida y evaluada adecuadamente para optar por nuevas intervenciones y guías de tratamiento médico. Por consiguiente, la enseñanza y actualización en ciencias básicas para el personal de salud debe considerarse prioritario, puesto que la dinámica de concepción en el tratamiento médico está cambiando desde la medicina curativa (diagnóstico y tratamiento), medicina preventiva (intervención para evitar o retrasar la aparición de la enfermedad) a la medicina predictiva (identificación del perfil genético para un tratamiento personalizado).
\end{abstract}

El desarrollo de nuevas áreas de investigación de los sistemas biológicos ha sido agrupadas en las "ómicas". Solo citaremos cuatro áreas (genómica, proteómica, transcriptómica y metabolómica), las cuales, últimamente, están siendo consideradas en estudios de tipo "caso y control".

La genómica estudia a los genomas de los individuos, ello incluye la secuenciación y análisis del ADN, para identificar nuevos genes y variaciones dentro de los mismos (polimorfismos) y su comparación con secuencias genómicas de otras poblaciones. La proteómica estudia y compara cuali- y cuantitativamente el perfil de proteínas (proteoma) presentes en un conjunto de células o tejidos en la presencia o ausencia de una enfermedad. La proteómica, no solo se limita a analizar el resultado de la expresión génica, sino que también estudia las modificaciones postraduccionales que pueden sufrir las proteínas, así como la interacción entre ellas. La transcriptómica estudia y compara los ARN y micro-ARN presentes en una célula, identificando los genes que se están expresando en un momento dado y el rol modulador que cumplen el micro ARN. La metabolómica identifica y cuantifica todos los metabolitos presentes en una célula o tejido u organismo en un momento determinado. Como se puede apreciar, las "ómicas" son importantes como disciplinas en sí mismas, las cuales nos ayudaran a entender la homeostasis del ser humano en presencia o ausencia de enfermedad ${ }^{(1)}$.

Dentro de las "ómicas", la genómica ha tenido mayor desarrollo. El primer secuenciamiento de un ser humano en el año 1998 costó aproximadamente 100 millones de dólares. Hoy en día podemos obtenerlo por 5 mil dólares. Sin embargo, se espera que en los próximos años esto pueda seguir bajando a un precio más accesible de 500 o 100 dólares. Por lo tanto, para secuenciar un genoma las limitaciones económicas y técnicas son cada vez menores; sin embargo, ahora, el desafío para los investigadores y científicos es determinar la forma de leer, entender e interpretar el contenido de las secuencias con relevancia clínica y cómo es la interacción entre ellas para poder descubrir la patología de las enfermedades humanas ${ }^{(2)}$. Los análisis individualizados, basados en el genoma de cada persona, conducirán a una forma muy

\footnotetext{
Instituto Nacional de Salud. Lima, Perú.

MD Phd, editor invitado

Recibido: 27-11-15 Aprobado: 27-11-15
}

Citar como: Guio H. Hacia la medicina personalizada: implicancias de las ciencias básicas y las "ómicas” en la práctica clínica. [editorial]. Rev Peru Med Exp Salud Publica. 2015;32(4):629-32. 
poderosa de medicina predictiva. Seremos capaces de aprender sobre los riesgos de enfermedades futuras con base en los análisis del ADN. ${ }^{(3,4)}$ Médicos, enfermeras, consejeros genéticos y otros profesionales del cuidado de la salud podrán trabajar con las personas para concentrar los esfuerzos en iniciativas que mantengan la salud de un individuo en particular. Esto podría significar una dieta o un cambio en el estilo de vida, o podría significar vigilancia médica con una nueva generación de intervenciones, muchas de las cuales serán medicinas bastante más eficaces y precisas que las disponibles hoy en día. Por consiguiente, una futura historia clínica del paciente estará constituida de un examen médico, ficha sociodemográfica, secuenciamiento de su genoma, datos del estado dinámico de las células (transcriptómica, proteómica y metabolómica) y flora patogénica (microbioma) (1). De este análisis surgen preguntas pertinentes: ¿el personal de salud está preparado para la interpretación de estos análisis?, ¿se está difundiendo adecuadamente estos conceptos en el pregrado y posgrado en los programas académicos de ciencias de la salud?. La genética de poblaciones, evolución de patógenos y selección natural para el desarrollo de enfermedades son temas que debería integrarse en la formación de profesionales de la salud ${ }^{(5)}$.

Las implicancias clínicas de la genética y genómica cada vez son mayores, su uso ya no es limitado a la identificación y seguimiento de enfermedades genéticas en el recién nacido, la evolución de nuevas metodologías y subáreas como la genética de poblaciones y la evolución de los patógenos, nos hace reflexionar por qué la selección natural hace que el ser humano se torne vulnerable a las enfermedades. Estamos viviendo un momento importante en la historia de la genética médica, ya que por primera vez podemos estudiar todo el genoma de una persona en nuestra búsqueda para encontrar la causa de la enfermedad subyacente y/o la mutación en determinado gen, la cual tenga un impacto en la respuesta clínica al tratamiento, en términos de mayor o menor efectos adversos y/o mayor, menor o nula respuesta al tratamiento. El genoma humano fue presentado a la comunidad mundial en el año $2001{ }^{(6)}$, las implicancias de los estudios de genes y genomas en las poblaciones es aceptado y promovido por la Organización Mundial de la Salud en el 2007; México fue el primer país latinoamericano en estudiar su diversidad genética en el cual se encuentra prevalencia de algunas enfermedades según la ancestria del lugar donde se toman las muestras ${ }^{(7)}$. El Instituto Nacional de Salud del Perú mostró en noviembre de 2015 sus primeros resultados del estudio de la diversidad genética en la población peruana, en el cual se reporta un gran componente nativo andino y amazónico en los mestizos peruanos, además de reportar, por primera vez, 3,3 millones de variaciones genéticas. El estudio completo será publicado el 2016; sin embargo, el siguiente paso es estudiar a pacientes con diagnóstico de enfermedades prevalentes en Perú como cáncer, diabetes e incluso enfermedades trasmisibles como tuberculosis o hepatitis, para identificar qué variantes genéticas de las que se están reportando serian responsables no solamente del desarrollo de una enfermedad, sino también del grado de severidad. No todos los pacientes reaccionan de la misma manera para controlar el avance de la enfermedad. Estos hallazgos permitirán identificar el mejor tratamiento con menores efectos nocivos, evitando el deterioro del paciente.

El sistema de salud japonés, después de estudiar genes relacionados al metabolismo de drogas antituberculosas está planteando la personalización del tratamiento, puesto que la concentración final de la droga en el cuerpo y, por consiguiente la efectividad, es afectada por la genética de cada individuo. ${ }^{(8,9)}$ En el presente número se analiza el "Rol de la Farmacogenómica en el régimen de tratamiento de tuberculosis", en el cual se explica la importancia de conocer los genes y sus polimorfismos relacionados al metabolismo de la izoniacida y rifampicina para un eficaz tratamiento de la tuberculosis con bajos efectos adversos. Asi mismo, el Dr. Flores-Angulo de la Universidad de Carabobo en Venezuela reporta los hallazgos de las variantes alelicas del CYP2D6 asociadas a enzimas metabolizadoras en la poblacion venezolana.

El soporte económico es muy importante para los institutos especializados, universidades y centros de investigacion para desarrollar, validar y favorecer la aplicación de los hallazgos de las ciencias básicas en el campo clínico para la prevención y tratamiento de las enfermedades $\left(10,{ }^{11)}\right.$. La Dra. Mazzetti jefa del departamento de Neurogenética del Instituto Nacional de Ciencias Neurológicas en Perú, muestra la importancia del desarrollo e implementación de estudios en ciencias básicas en el área de neurogenetica, exponiendo la manera como los avances de estudios y nuevo conocimiento en la enfermedad de Huntington debiera ser trasladado a la práctica clínica (investigación translacional).

La búsqueda de un tratamiento adecuado y sin efectos adversos han hecho que se explore terapias con células madre, por poseer características como: autorrenovación (proliferación indefinida) y diferenciación (adquirir características y funciones especializadas del lugar de implantación). Adicionalmente, el beneficio de obtener células madre adulta sobre las embrionarias, descarta la posibilidad de rechazo por parte del paciente. Muchos estudios a nivel experimental han sido exitosos y han permitido su aplicación en la medicina regenerativa, inmunoterapia y terapia génica. Sin embargo, en algunas áreas no hay estudios concluyentes y exitosos 
para su aplicación en el ser humano, por consiguiente, los investigadores y clínicos debieran conocer el desarrollo científico y las consideraciones éticas antes de promover su aplicación. El Dr. Amiel explica las limitaciones y oportunidades que se encuentran durante el desarrollo e implementación de esta área en el Perú. Los estudios genéticos en cáncer también deberían personalizarse. Muchas mutaciones encontradas en las tumoraciones son aquellas que estuvieron presentes desde el nacimiento y no son las responsables del cáncer. Actualmente, los estudios deberían enfocarse en estudiar el tejido con presencia y ausencia de cáncer del mismo paciente para establecer las mutaciones que son propias de la enfermedad ${ }^{(12)}$.

El uso de fitoquimicos a nivel experimental en cáncer también está teniendo un gran desarrollo en el campo de quimioprevención y tratamiento. (13) En el presente número se reporta dos estudios del efecto inmunomodulador de la Uncaria tomentosa (uña de gato). El primer reporte lo hace el Dr. Lozada en ratones con melanoma, mientras que el Dr. Nuñez evalúa el efecto en pacientes con cáncer de mama.

Sin duda, el desarrollo de las ciencias básicas es un compromiso multidisciplinario, por consiguiente, el salto cualitativo a un sistema de salud en términos de mejores condiciones tecnológicas y conceptuales favorecerá el desarrollo de una medicina más predictiva y personalizada. Todavía hay un $95 \%$ en el genoma que se cataloga como no codificante o no cumple una función, cuando en realidad todavía no entendemos o encontramos cómo medir los productos biológicos de estas regiones. EI ADN no está flotando, está envuelto en unas proteínas (histonas) las cuales ejercen una interacción con el ADN, éstas proteínas sufren modificaciones químicas influenciadas por el ambiente (epigenética) las cuales activaran o silenciaran la expresión de un gen. Estos cambios (metilaciones) pueden ser reversibles. Actualmente, ya se existen fármacos que reparan los patrones epigenéticos.

Finalmente, después del descubrimiento del ADN por Watson y Crick creímos que todo estaba resuelto. Ahora sabemos que hay otros sistemas complejos e interacciones dentro del ADN que hace que el individuo mantenga la resistencia o susceptibilidad a ciertas enfermedades. Los estudios de diversidad genética de cada población aunado a estudios de expresión génica en las enfermedades más prevalentes en un país nos ayudarán a elegir la mejor opción diagnóstica y terapéutica para obtener un mejor pronóstico con limitados efectos adversos.

\section{REFERENCIAS BIBLIOGRÁFICAS}

1. Topol EJ. Individualized medicine from prewomb to tomb. Cell. 2014 Mar 27;157(1):241-53. PubMed PMID: 24679539. Pubmed Central PMCID: 3995127.

2. Wang J, Liao J, Zhang J, Cheng WY, Hakenberg J, Ma M, et al. ClinLabGeneticist: a tool for clinical management of genetic variants from whole exome sequencing in clinical genetic laboratories. Genome medicine. 2015;7(1):77. PubMed PMID: 26338694. Pubmed Central PMCID: 4558641.

3. Veltman JA, Lupski JR. From genes to genomes in the clinic. Genome medicine. 2015;7(1):78. PubMed PMID: 26221187. Pubmed Central PMCID: 4517556.

4. Beaudet AL. Global genetic carrier testing: a vision for the future. Genome medicine. 2015;7(1):79. PubMed PMID: 26221188. Pubmed Central PMCID: 4517364.

5. Nesse RM, Bergstrom CT, Ellison PT, Flier JS, Gluckman P, Govindaraju $\mathrm{DR}$, et al. Evolution in health and medicine Sackler colloquium: Making evolutionary biology a basic science for medicine. Proceedings of the National Academy of Sciences of the United States of America. 2010 Jan 26;107 Suppl 1:1800-7. PubMed PMID: 19918069. Pubmed Central PMCID: 2868284.

6. Venter JC, Adams MD, Myers EW, Li PW, Mural RJ, Sutton GG, et al. The sequence of the human genome. Science. 2001 Feb 16;291(5507):130451. PubMed PMID: 11181995.

7. Silva-Zolezzi I, Hidalgo-Miranda A, Estrada-Gil J, Fernández-López JC, Uribe-Figueroa L, Contreras A, et al. Analysis of genomic diversity in Mexican Mestizo populations to develop genomic medicine in Mexico. Proceedings of the National Academy of Sciences of the United States of America. 2009 May 26;106(21):8611-6. PubMed PMID: 19433783. Pubmed Central PMCID: 2680428.

8. Azuma J, Ohno M, Kubota R, Yokota $S$, Nagai T, Tsuyuguchi K, et al. NAT2 genotype guided regimen reduces isoniazid-induced liver injury and early treatment failure in the 6-month four-drug standard treatment of tuberculosis: a randomized controlled trial for pharmacogenetics-based therapy. European journal of clinical pharmacology. 2013 May;69(5):1091101. PubMed PMID: 23150149. Pubmed Central PMCID: 3641305.

9. Kinzig-Schippers M, TomalikScharte D, Jetter A, Scheidel B, Jakob V, Rodamer M, et al. Should we use $\mathrm{N}$-acetyltransferase type 2 genotyping to personalize isoniazid doses? Antimicrobial agents and chemotherapy. 2005 May;49(5):17338. PubMed PMID: 15855489. Pubmed Central PMCID: 1087660.

10. Fang FC, Casadevall A. Lost in translation--basic science in the era of translational research. Infection and immunity. 2010 Feb;78(2):563-6. PubMed PMID: 20038540. Pubmed Central PMCID: 2812192.

11. Hobin JA, Deschamps AM, Bockman $\mathrm{R}$, Cohen S, Dechow P, Eng C, et al. 
Engagingbasic scientists in translational research: identifying opportunities, overcoming obstacles. Journal of translational medicine. 2012;10:72. PubMed PMID: 22500917. Pubmed Central PMCID: 3419626.

12. Jones S, Anagnostou V, Lytle K, Parpart-Li S, Nesselbush M, Riley DR, et al. Personalized genomic analyses for cancer mutation discovery and interpretation. Science translational medicine. 2015 Apr 15;7(283):283ra53. PubMed PMID: 25877891. Pubmed Central PMCID: 4442685.

13. Wang H, Khor TO, Shu L, Su ZY, Fuentes $\mathrm{F}$, Lee $\mathrm{JH}$, et al. Plants vs. cancer: a review on natural phytochemicals in preventing and treating cancers and their druggability. Anti-cancer agents in medicinal chemistry. 2012 Dec;12(10):1281-305. PubMed PMID: 22583408. Pubmed Central PMCID: 4017674.

\section{$h t t p: / / w w w . i n s . g o b . p e / r p m e s p$}

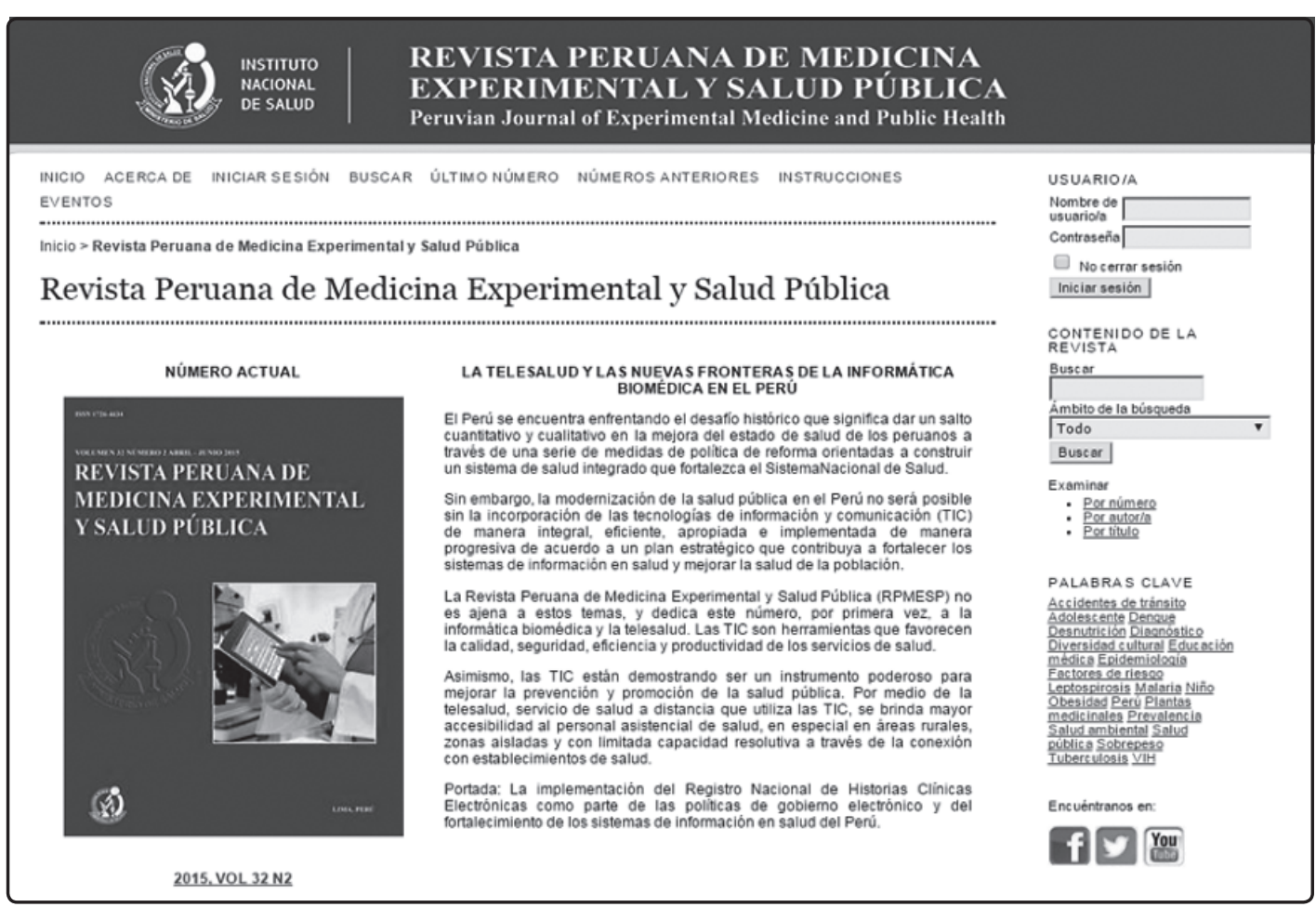

